Eurasian Journal of Physics and Functional Materials

$2021,5(2), 102-115$

\title{
An overwiew on corrosion behavior of steels in factory
}

\author{
H. Demiroren \\ Canakkale Onsekiz Mart University, Canakkale, Turkey \\ E-mail: hulya.demiroren@comu.edu.tr
}

DOI: 10.32523/ejpfm.2021050203

Received: 15.04.2021 - after revision

\begin{abstract}
In this study, corrosion behaviors of diffuser unit solution by which raw sugar is produced from minced sugar beets in sugar factories on St-37 low alloy steel and AISI 304, 304L, 316 austenitic stainless steel types were examined. Moreover, influence of heat treatment on corrosion resistance was investigated. Corrosion tests were performed using mass loss method. SEM-EDX, X-rays Diffraction, microhardness and surface hardness analyses of samples were carried out. As a result, it was determined that AISI $304 \mathrm{~L}$ and 316 steels have better corrosive resistance and heat treatment improves corrosion resistance.
\end{abstract}

Keywords: industrial factories, corrosion, stainless steel, heat treatment, SEM-EDS analysis.

\section{Introduction}

Corrosion is a significant problem with an economic dimension causing degradation because of chemical and electrochemical reactions entered by metals and alloys with their environment. Corrosion-caused losses are considerably high in industrial plants especially in chemistry and food industry. The industrial processing of sugar beet results in a lot of harmful effects on components of factory. The most important effect is corrosion. Importance of corrosion arises when sugar factories are evaluated in this aspect. Losses occurred due to the corrosion lead to a decline in capacity of a factory and decrease in occupational safety. Corrosion gains a great importance especially when it combines with mechanical factors, friction and cavitation impacts causing erosion.

One of the places most frequently experiencing corrosion event among units and plants of sugar factories is diffuser towers. Diffuser towers are the place 
where raw sugar juice is generated from sugar beet. Sugar in beets is included in solution in these towers. In addition, some impurities like ions, pectins, acid also pass are mixed with the solution. As a consequence, a liquid solution (sugar juice) acting as electrolyte in the diffuser is formed. Corrosion features of this electrolyte increase with high temperature $\left(>70^{\circ} \mathrm{C}\right)$ and low $\mathrm{pH}$.

Main reason of corrosion in diffusers in sugar juice extraction is the low $\mathrm{pH}$ values. The reason of this is acidification of diffusion feed water and formation of bacterial originated acid during the course of diffusion [1]. Lo et al. investigated the influences of solution treatment on the cavitation erosion and corrosion resistances of a CrMnNi austenitic stainless steel and determined that solution heat treatment degraded the corrosion and cavitation resistance of samples [2]. In the other study Cash et al. worked the low carbon steel. It was reported here for the corrosion of low carbon steel in mixed cane sugar juice (i.e. the juice leaving the extraction process) have included a study of the contribution of activation, concentration and resistance polarization to the cathodic reactions. Mathematical methods involving the generation and devolution of polarization curves were used to identify the corrosion reactions. The oxygen reduction reaction appears as the dominant cathodic reaction with both activation and diffusion polarization operative at the corrosion potential. The corrosion rate of the steel can be determined from the anodic polarization curve corrected for surface film resistance [3].

Corrosion rate of low alloy St37, AISI304, AISI304 L X2CR NiMoN225 alloy, GX7CrNiMoNb18.0 alloy aluminum and brass materials was determined in upper division sugar juice, lower division sugar juice and raw sugar juice medias in order to examine the effect of sugar juice solutions in different concentrations on diffuser material in the diffuser unit. It was seen from measurements that all media expect for raw sugar juice is corrosive. Electrodes prepared with AISI304, AISI304L steels displayed a low corrosion rate as 0.01-0.02 mm/year in all sugar juice medias. It was convinced that some parts of tower diffusers should be replaced in every 8 years due to the puncture. It was considered that punctures occur mostly in upper division part where $\mathrm{pH}$ is lower [4]. Corrosion in diffusers can be prevented with various coatings. Features and implementation technology of coatings were developed by Romenskii for an effective protection. Metal surface of diffuser should be coated with aluminum after cleaning (at the end of campaign). While aluminum is protected with its passive oxide layer, it also provides electrochemical (cathodic) protection for the system [5]. Jin et al. analysed the corrosion characteristic and risk in atmospheric tower mathematically using software. They determined, to protect of corrosion caused by temperature fluctuations in the tower, the quantity of lateral extraction should be adjusted in time when the processing load is changed. The corrosive medium in the feed can reduce the gas-phase mass flow rate in the atmospheric tower and they determined that this would change the gas content [6]. Sugar cane juice contains a high level of chloride (approximately 1300 ppm) [7]. Chlorides tend to become concentrated with sugar processing. According to Genie, use of stainless steel in tail section of the diffuser is the only precaution taken against corrosion even though some various dyes and coatings are recommended. Dyes 
and coatings are useful for prevention of corrosion between campaigns. However, they deteriorated and break down so rapidly during the time of beet processing [8]. Durmoo et al. investigated the behavior of stainless steel AISI 304L exposed sugar cane juice. As a result, they declared that uniform corrosion is low and depending on $\mathrm{pH}$ of sugar juice. They determined pitting corrosion but asked "this pitting corrosion is it chemical and electrochemical result or a biochemical result or a coupling of the two mechanisms?" [9]. Fuji et al. [10] investigated mechanical criteria for the nucleation of intergranular stress corrosion cracking (IGSCC) in thermally-sensitized type 304 austenitic stainless steel. They applied solution heat treatment to their sample. But they did not compare to unheated samples. In other paper, it was reported effect of heat treatment process on hot corrosion resistance of Fe25Ni-18Cr-1Mo-2 W-1Nb-cast austenite stainless steel [11]. Kumar et al. [12] Microstructure and corrosion behavior of warm deformed 316LN stainless steel was investigated in primary water reactor (PWR) environment by electrochemical corrosion test methods and SEM. They reported that heat treatment after deformation increases the pitting resistance of $316 \mathrm{~L}$ stainless steel in PWR environment. Grain size and residual strain on the pitting corrosion is still debatable and needs to be critically investigated. It was reported that the carbon concentration has not noticeable effect on pitting corrosion of austenitic stainless steel [13].

In this study, corrosion resistance of St37 steel and a group of austenitic stainless steel was determined. Besides effect of heat treatment on corrosion behavior was investigated. In this study, St37 steel, which is used as the basic building material of factories in industrial life, and a group of austenitic stainless steel, which is used in corrosive conditions that this steel cannot withstand, has been investigated. It was not found that such a comparison was systematically made in the same study. In addition, this work was carried out to solve a real problem rather than to make scientific progress.

\section{Experimental method}

St37 low alloy steel and AISI 304, AISI 304L and AISI 316 austenitic stainless steel group were used in this study. Experiment samples were prepared by cutting in $10 \mathrm{~mm} \times 10 \mathrm{~mm} \times 2 \mathrm{~mm}$ dimensions. Chemical analysis of samples is given in Table 1.

Sample S1 is the main material used in manufacturing of diffuser towers in sugar factories and on which austenitic stainless steels are cased (welded) for protection of corrosion. Sample S2 is the most commonly used austenitic stainless steel as a protective coating element since it is resistant against corrosion and cheap. Samples S3 and S4 are the materials accepted as more resistant in corrosive environments compare to sample S2.

Samples were examined in two groups as unheated and exposed to heat treatment. First group samples were not exposed to heat treatment, namely they are under production conditions, in other words, as out of the casting. Second group samples were exposed to heat treatment in $1100^{\circ} \mathrm{C}$ for 10 minutes under 


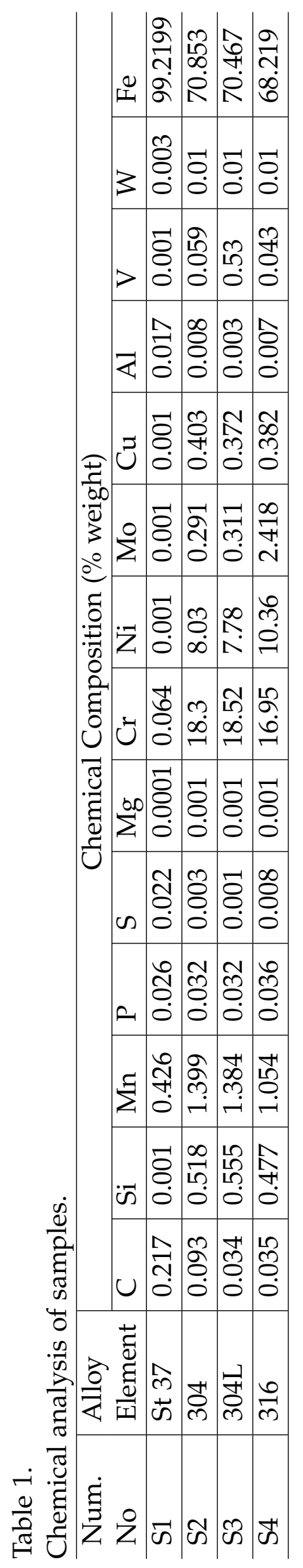


vacuum furnace and cooled in water. The tests were performed under air. SEM images of samples were monitored with LEO 440 brand electron microscope.

Corrosion experiments were performed with gravimetric method by using corrosion (coupon) monitoring test.

Samples were weighed by cleaning before and after the test. Cleaning was made according to standard method end of the test.

Liquid solution required to create the corrosion environment was received from upper division of tower diffuser in sugar factory. Because, it is the point where acid poured in the tower for $\mathrm{pH}$ balance enters and is in highest density. Explanations of factory officials and literature search have also evidenced that receiving solution from this point is right.

Specifications of the sugar juice received from upper division tap of the tower diffuser were tested in Sugar Factory laboratory and analysis results are as follows:

Temperature $=70^{\circ} \mathrm{C}$.

Polar Sugar (polarization value of a sugared solution read in saccharemeter according to ICUMSA method is called as polar sugar $)=0.43$.

Purity (The value obtained by multiplying the ratio between polar sugar amount read in saccharemeter (P\%) according to ICUMSA method and Brix amount read in refractometer $(\mathrm{S} \%)$ with 100 is called as the purity of that solution. It is indicated with $Q$.) $=0.72$.

Conductivity (Electricity conductivity of an impure sugared solution is known as concentration measure of ionic salts included in the solution.) $=14.23$.

It is a measure of saccharose amount in pure saccharose solution) $=0.6$ and $\mathrm{PH}=5.9$.

After recording their initial weights, samples were exposed to corrosion in a digital incubator in which the experiment solution exists and beakers were placed for periods of 2, 4, 6 and 8 hours. Incubator temperature was fixed in $70^{\circ} \mathrm{C}$. Weight determinations were made by weighing in XB220A PRECISA brand scale with $10^{-4} \mathrm{mg}$ sensitivity. Samples were received from the environment at the end of planned hours $(2,4,6$ and 8$)$ were washed with pure water and their final weights were measured after keeping them wait in desiccator about 1 hour. And then, mass loss records were detected, area calculations were made and finally corrosion rates were calculated.

\section{Results and discussion}

\section{Metallographic Investigations}

SEM photography, EDX analysis and X-rays diffraction of sample S1 is given in Figure 1a. The structure is $\alpha$-iron crystal species and the main structure is ferrite phase. Microstructure is composed of pearlite $\left(\alpha+\mathrm{Fe}_{3} \mathrm{C}\right)$ diffused between ferrite structures. It is considered that white large areas are ferrite, black areas are pearlitte. The reason why pearlite amount is greater, is due to the great amount of cementite $\left(\mathrm{Fe}_{3} \mathrm{C}\right)$ in the structure [14].

It is seen that particle size of the sample exposed to heat treatment decreased 

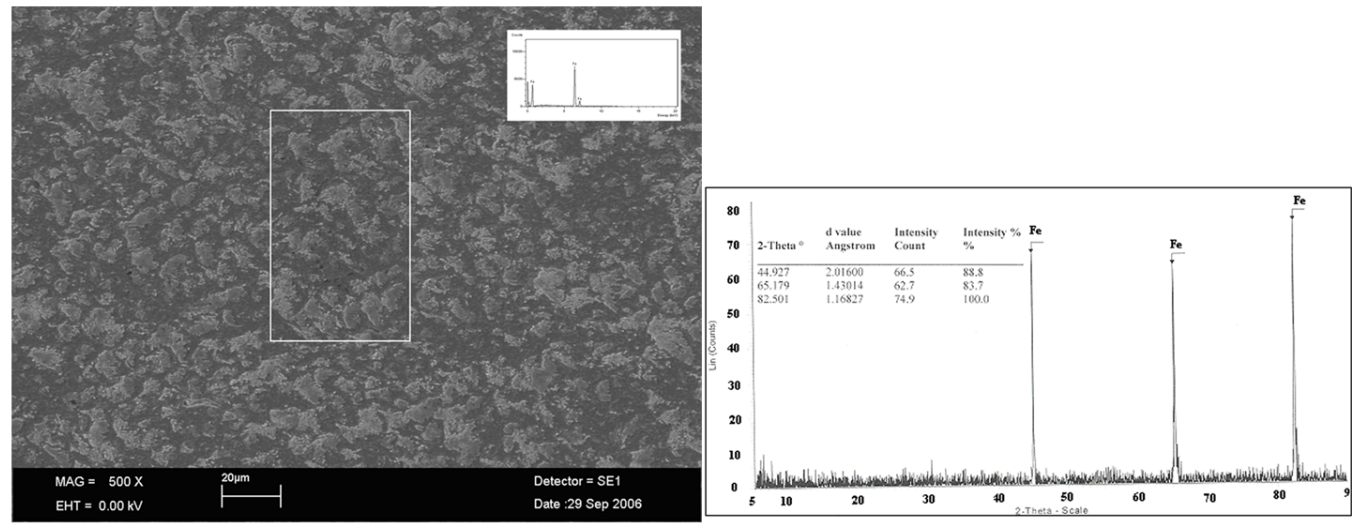

(a)
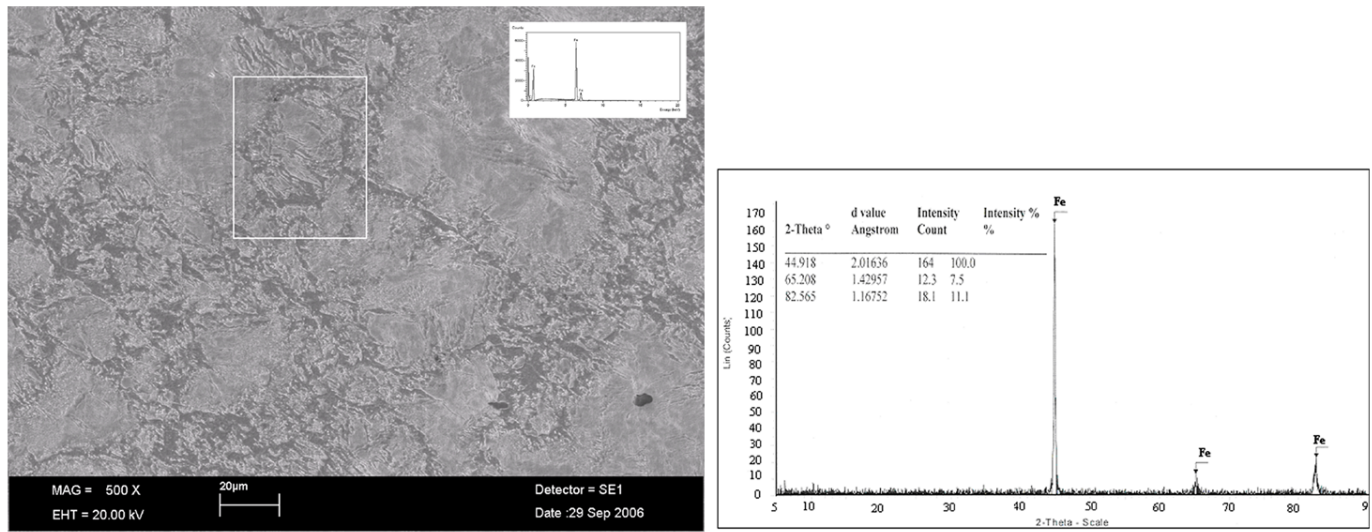

(b)

Figure 1. SEM photography (X500), EDX analysis, X-rays diffraction of a-untreated sample b-treated sample S1.

while looking at microstructure photographys in Figure 1b. To been decreased of particle size can be possible with re-crystallization happened with heat treatment. While the untreated sample was composed of considerably coarse particle sizes, treated sample is composed of small particle sizes. In other words, heat treatment has enabled the microstructure to be fine-grained. Moreover, it is also seen that main phase ferrite is denser and pearlite dispersion is gradually decreasing. The reason of increase in ferrite is that cementite was dissolved with heat treatment [14].

Main structure is austenitic $(\gamma-\mathrm{Fe})$ phase. However, $\mathrm{M}_{23} \mathrm{C}_{6}$ carbides exist in especially particle borders in the structure. Although it seems that no carbon exists as seen in EDX analysis (Table 2), actually carbon exists but in form of a compound in the area where point analysis is received [14]. SEM photography, EDX analysis and X-rays diffraction of untreated sample S2 are given in Figure 2a.

SEM photography, EDX analysis and X-rays diffraction of treated sample S2 are given in Figure $2 \mathrm{~b}$. Main structure is austenitic phase. $\mathrm{M}_{23} \mathrm{C}_{6}$ carbides were substantially dissolved in particle borders as a result of homogenizing heat treatment. It is seen from Figure $2 b$ that carbide dispersion in the structure decreased due to the impact of heat treatment. As seen from the results of EDX analysis, carbon which is an intermetallic phase decomposed from its compounds and became visible alone in form of a phase as a result of homogenizing process in $1100^{\circ} \mathrm{C}[14]$. 


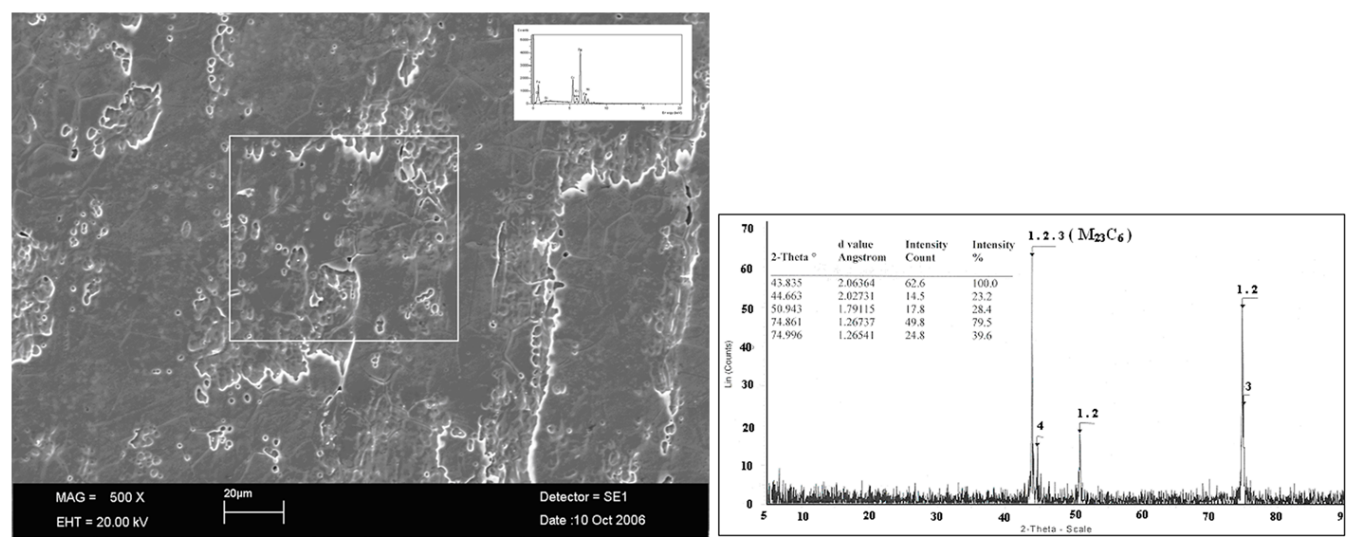

(a)
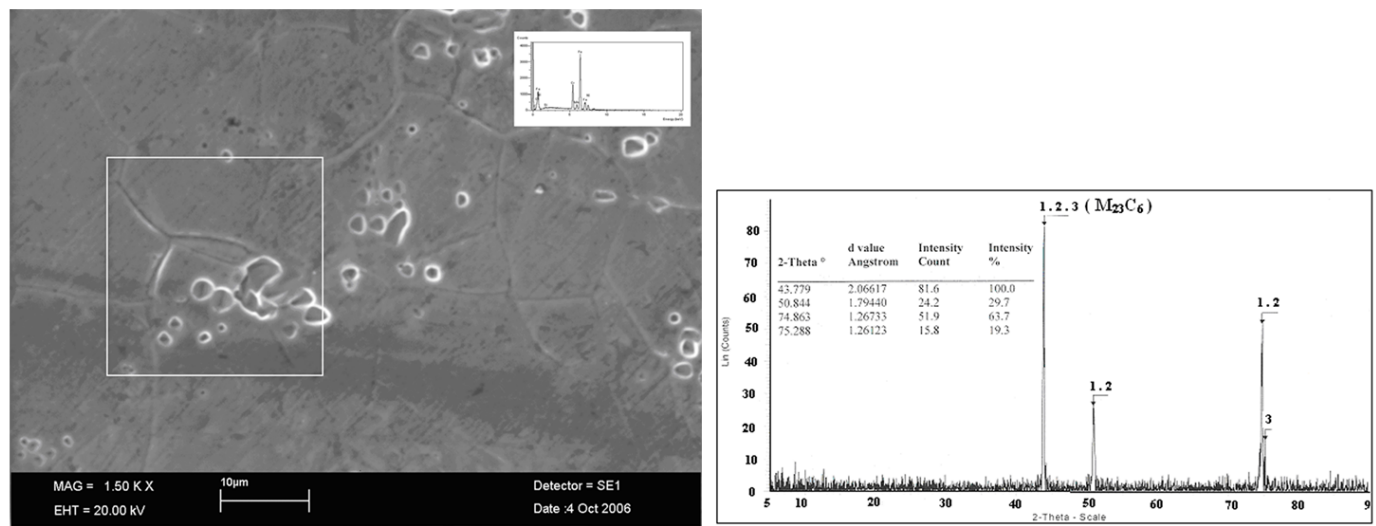

(b)

Figure 2. SEM photography (X500), EDX analysis, X-ray diffraction of a-untreated b-treated sample S2.

Main structure is austenitic phase. $\mathrm{M}_{23} \mathrm{C}_{6}$ carbides appear in especially particle borders in the structure in SEM photography of sample. As seen SEM photography, particle size of carbides in the structure of AISI 304L is larger and has a lower density compare to the particle size of carbides in the structure of AISI 304 even though photographies are in the same zoom. It can be said that this situation results from low carbon content of the alloy [14]. SEM photography, EDX analysis and X-rays diffraction of untreated AISI-304L austenitic stainless steel sample are given in Figure 3a.

SEM photography, EDX analysis and X-rays diffraction of treated S3 sample are given in Figure $3 \mathrm{~b}$. Main structure is austenitic phase. $\mathrm{M}_{23} \mathrm{C}_{6}$ carbides were substantially dissolved in particle borders as a result of homogenizing heat treatment. As seen from SEM photography, carbide dispersion in the structure decreased and there appears rare carbides gone away from particle borders due to the impact of heat treatment [14].

SEM photography, EDX analysis and X-rays diffraction of untreated AISI 316 austenitic stainless steel sample are given in Figure 4a. Main structure is austenitic phase. While the ratio of $\mathrm{M}_{23} \mathrm{C}_{6}$ carbides is high, it is appeared rarely the ratio of MoC carbides is low [14].

$\mathrm{M}_{23} \mathrm{C}_{6}$ carbides dissolved in a low ratio, MoC carbides were formed by combining with molybdenum element in which carbon is lower than chromium instead of them after homogenizing heat treatment. Carbide dispersion has lower density compared to untreated sample, carbides are observed as separated from 

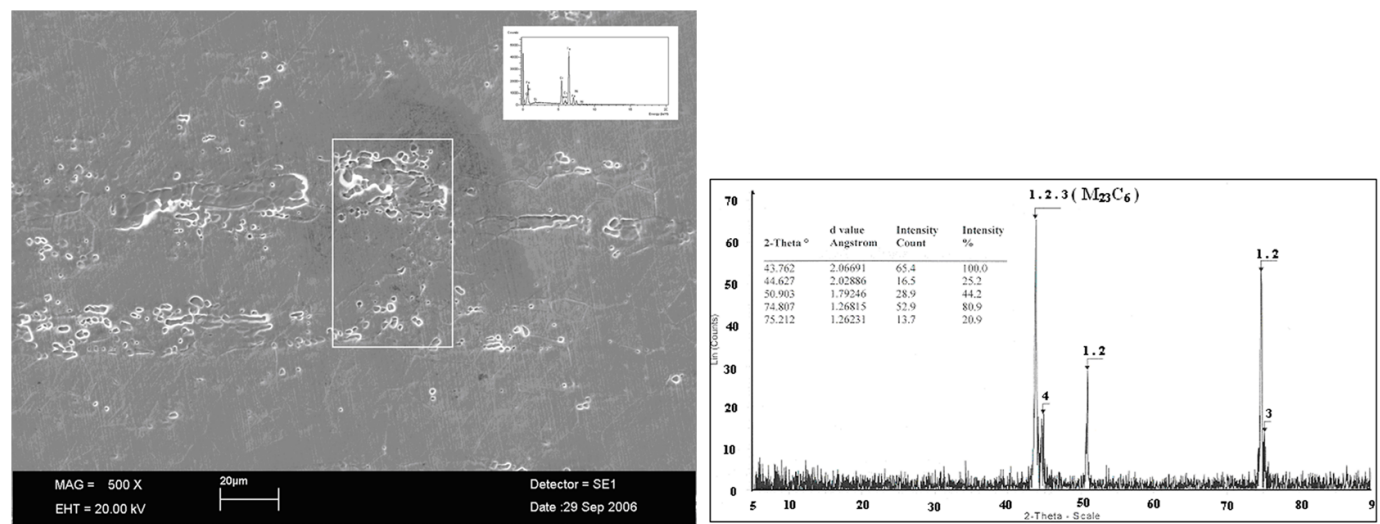

(a)
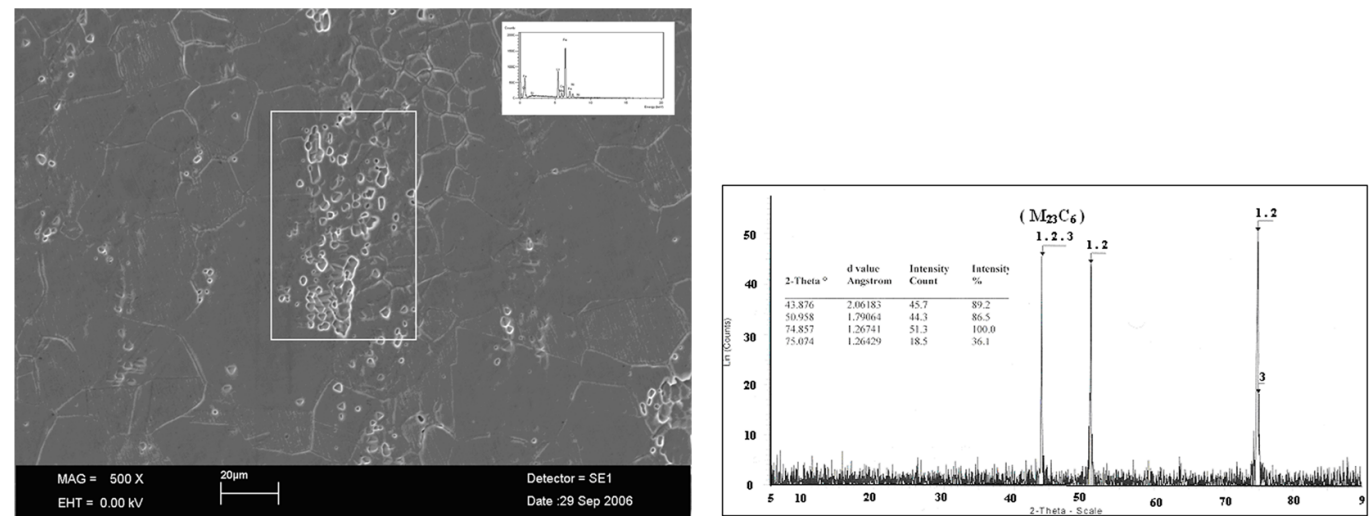

(b)

Figure 3. SEM photography (X500), EDX analysis, X-rays diffraction of a-untreated b-treated sample S3.

each other and dissolved some more [14]. SEM photography, EDX analysis and $\mathrm{X}$-rays diffraction of sample treated $\mathrm{S} 4$ are given in Figure $4 \mathrm{~b}$.

Brinell hardness values received from surface of samples are indicated in Table 2. Hardness of untreated samples risen by the increase in alloy ratios and diversities. It is though that these results from each alloy element additives in stainless steel samples and carbides existing in the structure. Typically, austenitic steels are processed hardly compared to low carbon and low alloy steels [15]. Higher degree of resistance and ductility, high deformation hardening tendency and low thermal conductivity compare to low carbon and low alloy steels are considered as reasons for why austenitic stainless steels are processed hardly [16]. Samples S2 and S3 have harder surface due to the chromium carbides in the structure, since both samples are more convenient for carbide formation compared to sample S4.

Homogenizing process caused an increase in hardness of especially sample S1. Reason of this increase in treated sample compared to untreated sample S1 is re-crystallization occurred with heat treatment and emergence of microstructure having a smaller particle size after this. Accordingly, it is possible to say that hardness and resistance will increase by the decline in particle size.

Table 2.

Brinell hardness values of samples. 

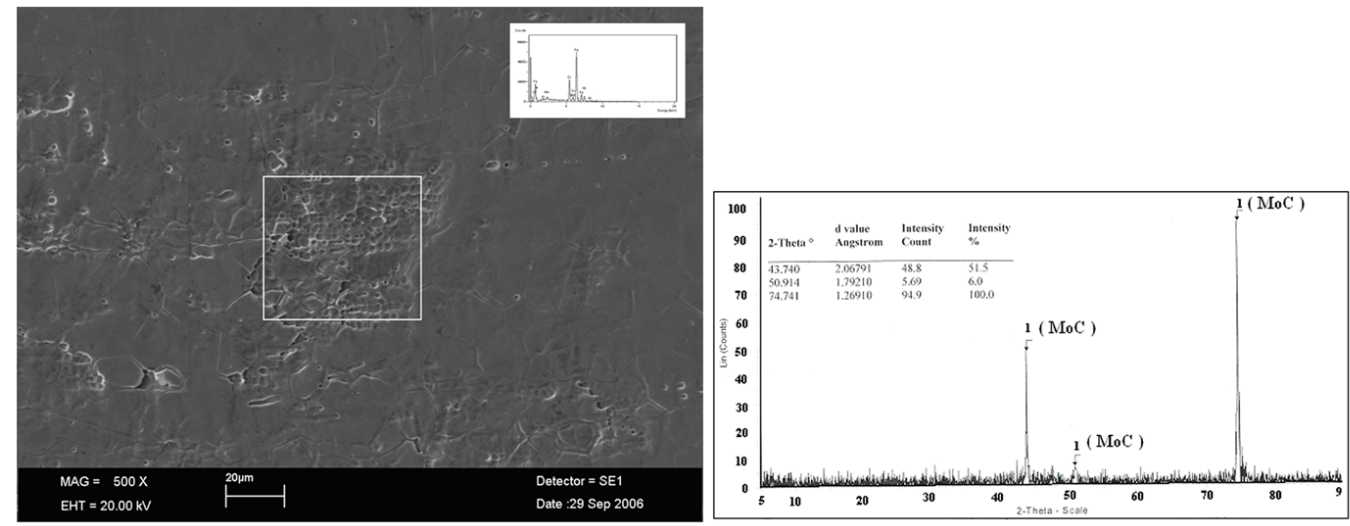

(a)
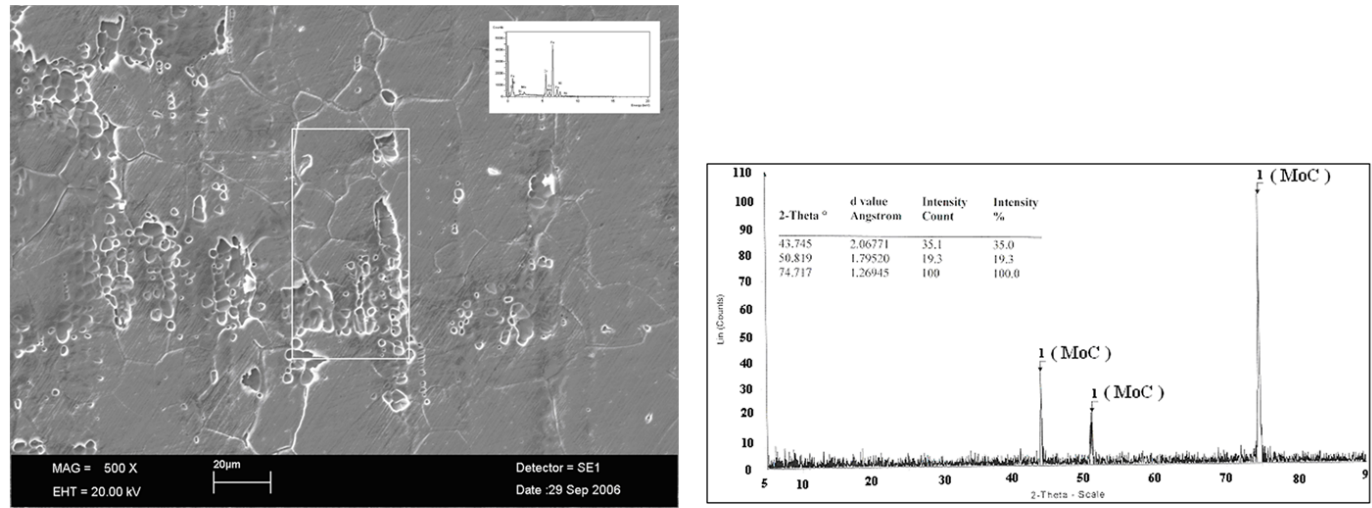

(b)

Figure 4. SEM photography (X500), EDX analysis, X-rays diff. of a-untreated b- treated sample S4.

\begin{tabular}{c|l|l|l|l}
\hline \multirow{2}{*}{$\begin{array}{c}\text { Heat } \\
\text { Treatment } \\
\text { Status }\end{array}$} & \multicolumn{5}{|c}{ Surface hardness (BRN)(3000 kgf-10mm ball) } \\
\cline { 2 - 5 } & S1 & S2 & S3 & S4 \\
\hline Untreated & 112 & 135 & 135 & 130 \\
\hline Treated & 220 & 135 & 128 & 117 \\
\hline
\end{tabular}

It is thought that the decline in the hardness of sample S3 probably resulted from carbides dissolved and decreased with heat treatment and increasing nickel rise as it is understood from EDX point analysis data table. While rise in nickel increases impact energy (in-depth hardness) it decreases surface hardness [17]. The reason of decline in hardness of sample S4 causes from the decrease in carbon, molybdenum and carbides in the structure with heat treatment.

Microhardness values measured from the matrix as Vickers are given in Table 3. $200 \mathrm{~g}$. load is used while measuring the micro hardness of samples, hardness value obtained is the average of 3 measurements taken for each sample.

Table 3.

Microhardness values of samples.

\begin{tabular}{c|l|l|l|l}
\hline \multirow{2}{*}{$\begin{array}{c}\text { Heat } \\
\text { Treatment } \\
\text { Status }\end{array}$} & \multicolumn{5}{|c}{ Matrix hardness (Micro hardness HV) } \\
\cline { 2 - 5 } & S1 & S2 & S3 & S4 \\
\hline Untreated & 312 & 367 & 539 & 341 \\
\hline Treated & 383 & 470 & 590 & 391 \\
\hline
\end{tabular}


When microhardness measurements are examined, the main reason of low hardness in untreated samples can be explained with the reason that microhardness is received from the matrix instead of particle borders. If the microhardness was received from grain boundaries, the load applied can be coincided to the carbides and consequently hardness can determined high values. It is possible to say that resistance will also increase in parallel to the increase occurred in hardness. The reason of this increase occurred in hardness and so in resistance if the formation of smaller microstructure with dynamic recrystallization in samples exposed to heat treatment [18]. Since the hardness was received from the matrix, increase in the hardness of treated samples can be explained with the dispersion of $C$ and $C r$ elements of which in particle borders $\left(M_{23} C_{6}\right)$ were released homogenously after heat treatment in the matrix.

Corrosion test results of untreated samples

Corrosion rate graphs of the untreated samples are given in Figure 5.

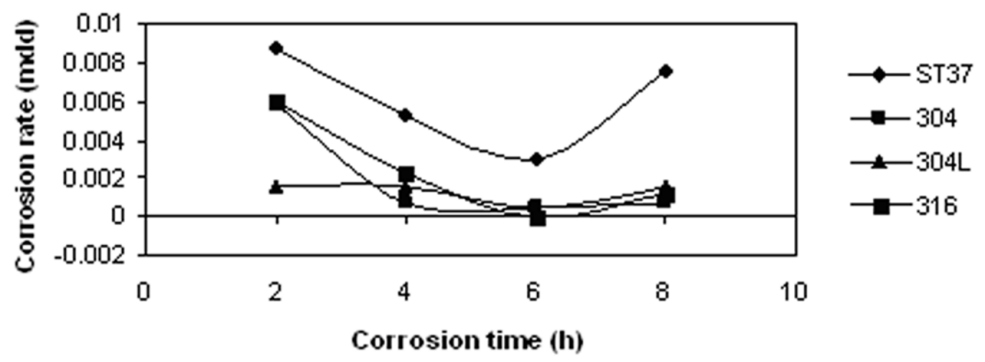

Figure 5. Corrosion rate of untreated samples (mdd).

The corrosion rates of untreated samples are seen in Figure 5. St 37 low alloy steel has the highest corrosion rate.

While the untreated sample displays a high corrosion rate during the first 2 hours of experiment period, displays a stable decline between 2-4 hours periods. The corrosion rate decreased after 2 hours. This is considered to be due to covering the surface the corrosion products occurring in the first 2 hours as seen in Figure 6. Iron oxide particles broken from the surface as a result of disruption of the protective layer in time increased causes pitting corrosion.

Corrosion rate of sample S2 is high in the first 2 hours of experiment period. It was observed that it decreased by means of the protective layer formed on sample surface end of $4^{\text {th }}$ hour (Figure $6 \mathrm{~b}$ ). If the products resulting from corrosion accumulate, a protective film forms on metal surface and so corrosion rate decreases [19]. Some raises may occur at corrosion rate when dissolubility rate of protective layer is higher than its formation rate.

It is thought that remainders which were formed on the surface of untreated sample S3 as a result of rapid corrosion in the first 2 hours of the experiment covered the surface and protected sample S3. This situation can be explained with the fact that dissolubility rate of protective film (Figure 6c) is higher than its formation rate [13].

Additive ratio of chromium element is declined by adding molybdenum in AISI 316 austenitic stainless steels. The less chromium increases dissolubility of carbon and slows down the kinetic of $\mathrm{M}_{23} \mathrm{C}_{6}$ carbide. Carbide formation process in which molybdenum will cause inter-particles corrosion by replacing 


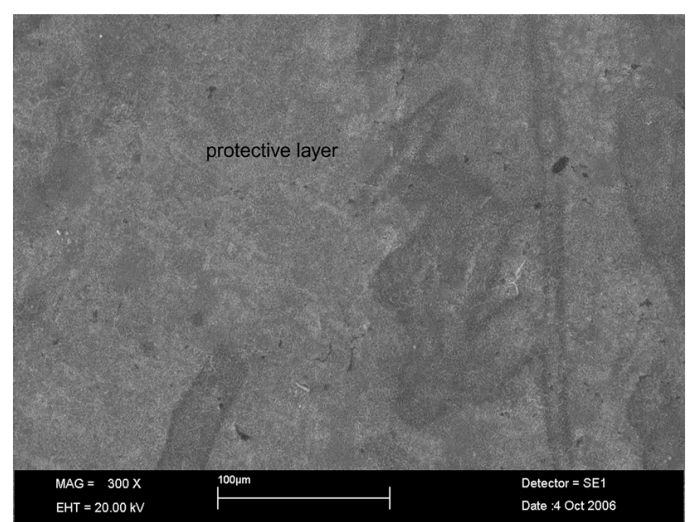

a

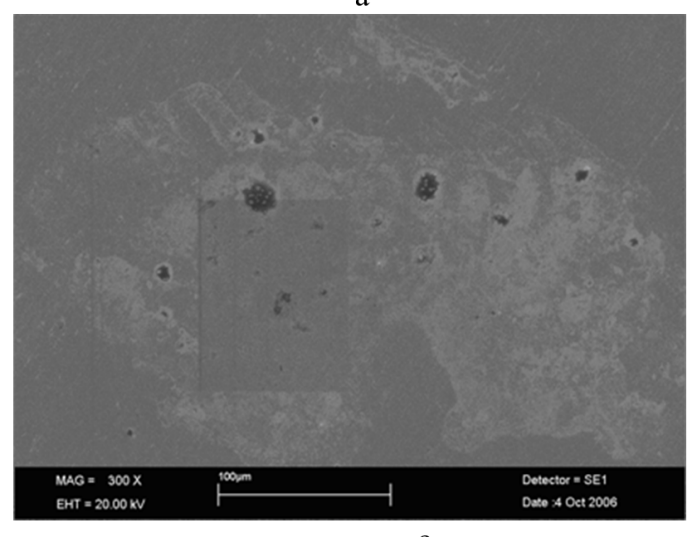

c

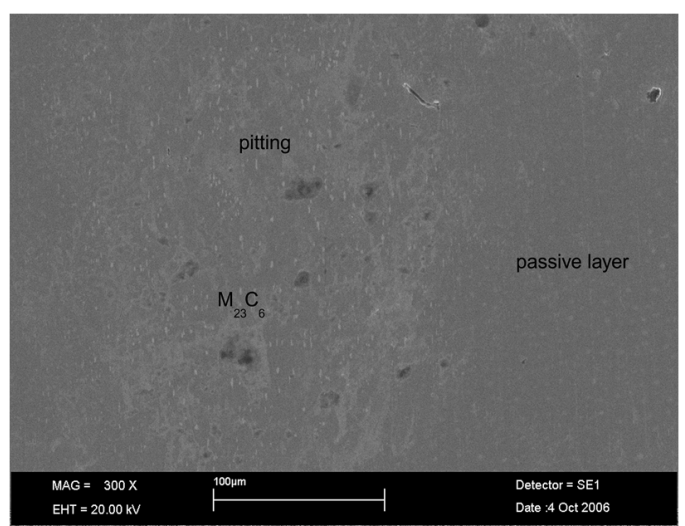

b

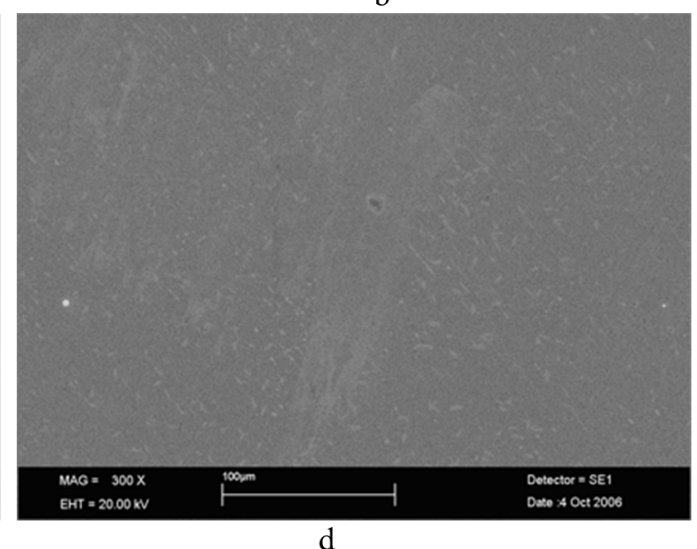

Figure 6. SEM photographies (X300) of untreated a-St 37 b-AISI 304 c-AISI 304L d-AISI 316 stainless steel samples exposed to corrosion for 4 hours (X300).

chromium, extends by the decline in chromium ratio. The most significant effect of molybdenum alloying is to slow down the chromium and carbon diffusion in austenitic phase about ten times. Moreover, molybdenum containment of carbide causes molybdenum consumption in particle borders [20]. It is seen from Figure $6 \mathrm{~d}$ that MoC carbide of untreated sample S4 exposed to corrosion for 4 hours and molybdenum in particle borders declined.

Corrosion test results of treated samples

Corrosion rate graphs of samples are given in Figure 7.

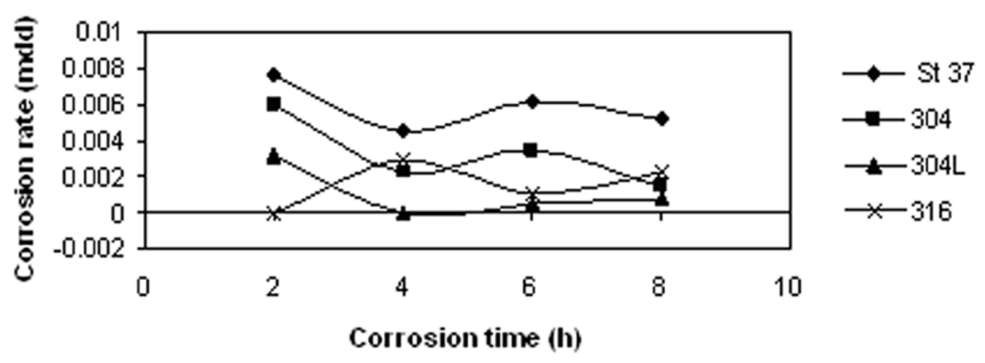

Figure 7. Corrosion rate of treated samples (mdd).

It is understood that the highest value in terms of corrosion according to heat treatment conditions is in the sample without heat treatment as seen from Figure 7 .

It is seen from photographies of both samples in Figure 6 and Figure 8 that particle size decreases with heat treatment. Materials with small particle size 
have some advantages like high resistance, high toughness and high fatigue life. Since the decrease in particle size increases resistance, it is possible to say that it will also increase corrosion resistance [21].

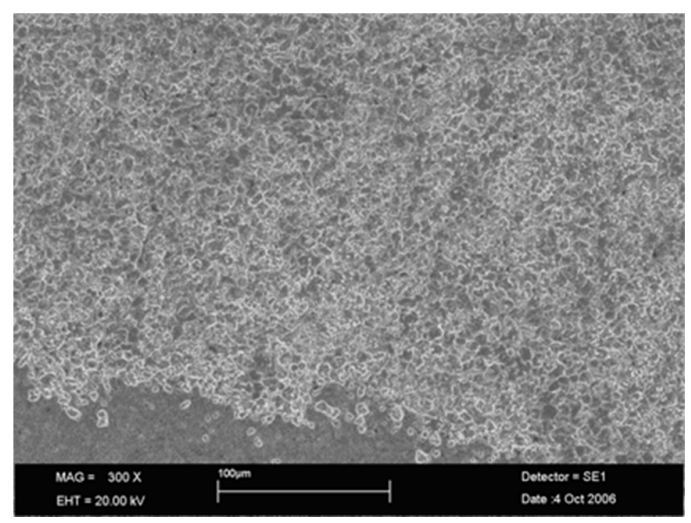

a

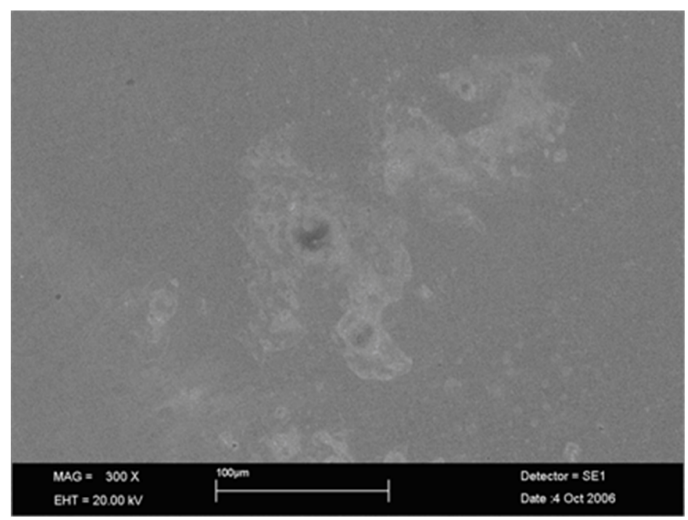

c

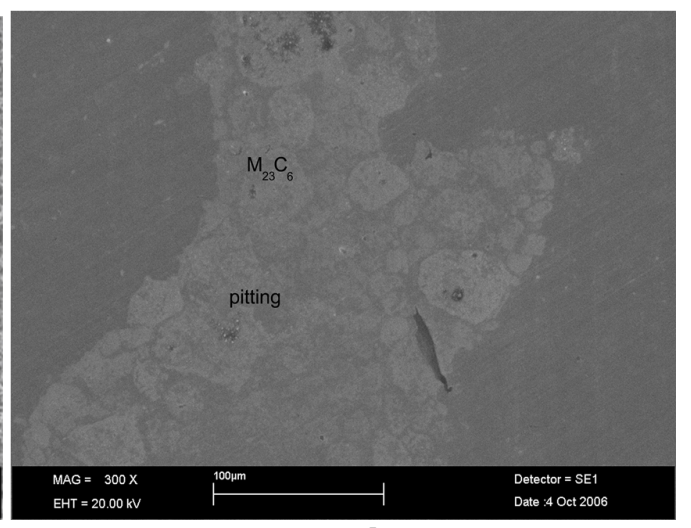

b

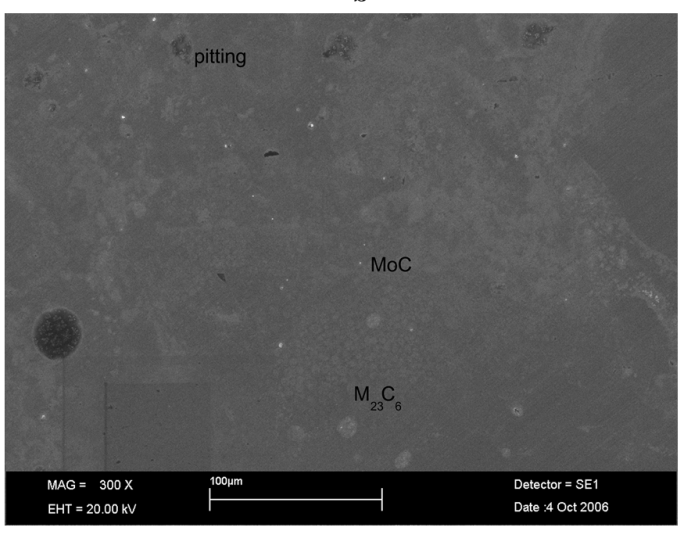

d

Figure 8. SEM photographies (X300) of treated samples a-St 37; b-AISI 304; c-AISI 304L; d-AISI 316 steel exposed to corrosion for 8 hours.

As seen from Figure 7, corrosion rate is high in samples S1 and S2, because protective layer did not form at the beginning. Then, corrosion rate decreased because the protective layer is formed. Protective layer disintegrated by increasing heat and corrosion rate increase. However, while this increase continues for untreated sample, it decreases reforming of the protective layer for treated sample, as seen in Figure 8.

The reason of high corrosion rate at treated sample S2 may be interrelated to not dissolve $\mathrm{M}_{23} \mathrm{C}_{6}$ carbides completely. This means that the period of homogenization process was not sufficient for this sample [13].

While corrosion rate of treated sample started to decline at the end of $6^{\text {th }}$ hour untreated sample started to increase only a little. This case can be attributed to the dissolving of carbides even a little although it was unsufficient took their effect at the end of this hour and the protective layer disintegrated was reformed for both samples at the end of $4^{\text {th }}$ hour. It can be said that treated and untreated samples have approximately the same corrosion rate at the end of $8^{\text {th }}$ hour.

Corrosion rate of treated steel sample S3 is higher than the corrosion rate of untreated sample S3 in the first 2 hours. It can be said that this case resulted from the fact that protective layer did not formed in this sampleat beginnig. Main reason of the decline in corrosion rate in following periods is that carbides in the 
structure were dissolved with heat treatment. In this case, chromium disengaged from carbon weakened with heat treatment re-strengths the regions weakened in terms of corrosion through forming chromium oxide by coming from inner regions of particles to particle borders and so naturally prevents the increase in weight loss [22].

Increases and declines are seen in the corrosion rate of treated sample S3 in time. This indicates that dissolution rate of the protective layer formed by chromium is equal to its formation rate. Homogenization treatment took the effect and corrosion decreased rapidly at the end of fourth hour. This case can be explained by the formation of protective layer in the surface. Formation rate of protective layer is higher than its formation rate [13]. Protective layer formed in the surface of treated sample S3 at the end of eight hours is seen in Figure 8.

According to corrosion rate graphs (Figure 6 and Figure 8), the sample having the best corrosion resistance is treated sample when AISI 316 steel samples are compared. While the lowest corrosion rate observes at treated sample in the first two hours of the experiment period, declines on one hand and increases in other hand were observed in both samples in following periods. The reason of this is that the protective layer formed decomposed on one hand and was formed on other. Corrosion rate increases when decomposition rate is higher than formation rate [13].

Mo element additive created a positive impact on corrosion resistance. This positive impact distinguishes itself better in treated sample (Figure 5). Because impact of corrosion declines with the decrease in the impact of existing $\mathrm{M}_{23} \mathrm{C}_{6}$ 's with homogenizing process [22].

\section{Conclusion}

It was determined that heat treatment improved corrosion resistance of samples due to both decline in particle size and dissolution of carbides.

Corrosion resistance as follows; AISI $316>$ AISI 304L >AISI 304> St 37. Corrosion resistance is better in AISI 316 and AISI 304L steels. Because it will form $\mathrm{M}_{23} \mathrm{C}_{6}$ carbides is low $(0.03 \%)$ in these steels due to their chemical structure. Moreover, molybdenum exists in AISI 316 steel, and it is also understood from former studies that this steel increases corrosion resistance.

According to experiment data, it can be said that diffuser solution austenitic samples will not create a great risk for stainless steel samples in terms of intergranular corrosion behavior provided that carbide formation temperature is not present. However, if austenitic stainless steels to be coated on St 37 low alloy steel are kept in waiting on $500^{\circ} \mathrm{C}-800^{\circ} \mathrm{C}$ range for a sufficient period during the course of tempering or welding processes, they will become sensitive towards intergranular corrosion. This type of corrosion continues without creating a change in appearance of the structure and weakens the material without noticing. This is so dangerous for sugar factories.

As a result of this study, stainless steels to be recommended in sugar factories diffuser units in terms of corrosion resistance in order of priorities are as follows; 
AISI 316, AISI 304L, AISI 304. Exposure of stainless steel to be selected to $1100^{\circ} \mathrm{C}$ homogenizing process by the producer firm after production is significant in terms of avoiding carbide formation during the carbide formation temperature. On the other hand, it can be said that St 37 is excessively affected from the solution even under normal conditions and its corrosion rate is high.

\section{References}

[1] S. Durmooa et al., Electrochimica Acta 54 (2008) 74-79.

[2] K.H. Lo et al., Wear 392-393 (2017) 159-166.

[3] G. A.Cash, D.P. Schweinsberg, G.A. Hope, Corrosion Science 30(6-7) (1990) 543-554.

[4] N. Karavaizoglu and M. Kabasakaloglu, $3^{\text {th }}$ Corrosion Syposium, Ankara (1998) 199-208.

[5] S.R. Kumar et al., Materials Todays 45 (2021) 1878-1881.

[6] H. Jin et al., Engineering Failure Analysis 122 (2021) 105283.

[7] G.A. Cash et al., Corros. Sci. 33(5) (1992) 751.

[8] G.V. Genie, Sugar Techn. 9 (1982) 119-270.

[9] S. Durmoo et al., Electrochimica Acta 54 (2008) 74-79.

[10] T. Fujii et al., Forces in Mechanics 3 (2021) 100013.

[11] M.S. Anwar et al., Materials Today: Proceedings 44 (2021) 3434-3438.

[12] V. Kumara et al., Materials Today: Proceedings 22(2020) 3347-3352.

[13] S.K. Pradhan et al., Corrosion Science 158 (2019) 108091.

[14] H. Demiroren, J. App. Electrochem. 39 (2009) 39:761-767.

[15] J. Paro et al., J. Mater. Process. Technol. 14 (2001) 119-130.

[16] 16 L.P. Karjalainen et al., Steel Res. Int. 79 (2016) 404-412.

[17] E. Zumelzu et al., J. Mater. Process. Technol 16 (2002) 250-255.

[18] K. Karacif and B. Inem, J. Fac. Eng. Archit. 16(1) (2001) 1-8.

[19] P. Refait et al., Corrosion Science 71 (2013) 32-36.

[20] R. Lackner et al., BHM 158(9) (2013) 364-370.

[21] M. Soleimani et al., Mater. Res. Express 7 (2020) 016522.

[22] J.R.S. Moreno and C.E.S. Junior, American Journal of Materials Science 4(5) (2014) 185-189. 